\title{
QOS - BASED RESOURCE ALLOCATION ALGORITHM FOR LONG TERM EVOLUTION (LTE) SYSTEM
}

\author{
Mohsen M. Tantawy ${ }^{1}$, Adly S. Tag ELdien ${ }^{2}$, and \\ Rokaia Mounir Zaki ${ }^{3}$ \\ ${ }^{I}$ Network planning Dept., National Telecommunication Institute, Cairo, \\ Egypt \\ ${ }^{2,3}$ Elec. Eng. Dept. faculty of engineering, Benha university, Shoubra, \\ Cairo, Egypt \\ e-mail:1'mohsen@nti.sci.ege-mail: 2adlymerg@yahoo.com \\ e-mail: ${ }^{3}$ rukaia.emam@feng.bu.edu.eg
}

(Received August 3, 2011 Accepted September 13, 2011)

\begin{abstract}
Long Term Evolution (LTE) has emerged as a comprehensive evolution of the Universal Mobile Telecommunications System (UMTS). In this paper, a Quality of Service (QoS)-guaranteed cross-layer resource allocation algorithm for multiclass services in LTE system is proposed. Numerical results demonstrate that the proposed algorithm can provide better behavior for QoS-based services than other previous resource allocation algorithms.
\end{abstract}

KEYWORDS: LTE; QoS; OFDM; Cross-Layer scheduling algorithms; GBR; Non-GBR.

\section{INTRODUCTION}

The goal of LTE is to provide a high-data-rate, low-latency and packet-optimized radio access technology supporting flexible bandwidth deployments [1], [2]. A broad range of multimedia applications with guaranteed quality of service (QoS) is expected to be provided by LTE. To ensure high spectral efficiency in the LTE cell while providing the required QoS, much more focus should be on dynamic packet scheduling [3].

The LTE air interface supports both frequency division duplex (FDD) and time division duplex (TDD) modes, each of which has its own frame structure. Subcarrier spacing is constant regardless of the channel bandwidth. Downlink and uplink transmission in LTE are based on the use of multiple access technologies: specifically, orthogonal frequency division multiple access (OFDMA) for the downlink, and singlecarrier frequency division multiple access (SC-FDMA) for the uplink [4], [5]. The advantage of OFDM is that; the radio channel is divided into many narrowband, lowrate, frequency-non-selective subcarriers, so that; multiple symbols can be transmitted in parallel and multiple user equipments (UEs) get assigned subcarriers or subsets of them in order to be served simultaneously. However OFDM was chosen as the signalbearer format because it is very resilient to interference.

It is also a modulation format that is very suitable for carrying high data rates one of the key requirements for LTE [6], [7]. In LTE, The configured classes have been specified in two categories of bearers, Guaranteed Bit Rate (GBR) and NonGuaranteed Bit-Rate (Non-GBR) bearers [8]. QoS Class Identifier (QCI) is an index 
that identifies a set of locally configured values for three QoS attributes: priority, delay and loss rate. QCI is signaled instead of the values of these parameters. The standard QCI classes are shown in table 1[9], [10]. When a connection (or bearer) is established between the UE and the LTE core network a QCI is specified. This defines whether the bearer is guaranteed bit-rate or not [11], [19]. Scheduling algorithms are responsible for selecting which UEs will have access to the system resources and with which configuration.

Adaptive Modulation and Coding (AMC) is the mechanism used for link adaptation to improve data throughput in a fading channel. This technique varies the downlink modulation coding scheme based on the channel conditions of each user [17]. Inside each subcarrier AMC is applied with three modulation schemes (QPSK, 16QAM and 64QAM) and variable code rates.

In order to exploit the advantages of OFDMA multiple access scheme and guarantee the QoS of different services with distinct traffic patterns and requirements, scheduling is importance in LTE. Scheduling algorithms are responsible for selecting which UEs will have access to the system resources and with which configuration [6], [7], [11]. Therefore, in this paper, we deal with downlink scheduling algorithms for capacity maximization in multiservice scenarios.

This paper is organized as follows: Section II presents an overview for the related works. Section III will establish the system model and analysis. In Section IV, the detailed cross-layer resource block allocation algorithm for LTE system is presented. In Section V, we investigated the performance of the proposed scheme and compare it with the traditional fixed and fair schemes. Finally, we conclude the paper in Section VI.

Table (1) Standardized QoS Class Identifiers (QCIs) for LTE [15].

\begin{tabular}{|c|c|c|c|c|}
\hline QCI & Resource type & Priority & Delay budget & Services \\
\hline 1 & GBR & 2 & $100 \mathrm{~ms}$ & VoIP. \\
2 & GBR & 4 & $150 \mathrm{~ms}$ & Video call. \\
3 & GBR & 5 & $300 \mathrm{~ms}$ & Streaming. \\
4 & GBR & 3 & $50 \mathrm{~ms}$ & Real time gaming. \\
5 & Non-GBR & 1 & $100 \mathrm{~ms}$ & IMS signaling. \\
6 & Non-GBR & 7 & $100 \mathrm{~ms}$ & Interactive gaming. \\
7 & Non-GBR & 6 & $300 \mathrm{~ms}$ & Applications with TCP: \\
8 & Non-GBR & 8 & $300 \mathrm{~ms}$ & browsing, email, file \\
9 & Non-GBR & 9 & $300 \mathrm{~ms}$ & download, etc. \\
\hline
\end{tabular}

\section{RELATED WORK}

In [18], the authors propose an adaptive proportional fair scheduling algorithm for LTE which adjusts the scheduling priority according to individual user's channel condition. This method gives more scheduling probability to the users who are under poor channel condition for a long period of time, and avoids the users whose channel 
conditions are favorable occupying too much resource. It enhances the fairness with a limited degradation of whole system throughput.

Authors of [13] have considered a flexible OFDMA wireless system, in which the fixed and fair allocation algorithms are explained. The results show the drawback of these algorithms for performance and fairness.

And in [14], the proposed cross-layer maximum weighted capacity (MWC) based resource allocation provides a much better QoS than maximum capacity (MC) and proportional fairness (PF) at a high total data arrival rate, while maintaining nearly the highest system capacity and costing a similar complexity.

\section{SYSTEM MODEL}

In this paper, an OFDMA system for LTE is considered. OFDM provides a physical basis for the multiple shared channels, where the total bandwidth $\mathrm{B}$ is divided into $\mathrm{N}$ subcarriers and each subcarrier have a bandwidth $B_{n}$ equal $B / N$. Let $n=\{1,2, \ldots, N\}$ denote the subcarrier index set.

LTE frames are $10 \mathrm{msec}$ in duration. They are divided into 10 subframes, each is $1.0 \mathrm{msec}$ long. Each subframe is further divided into two slots, each of $0.5 \mathrm{msec}$ duration. Slots consist of either 6 or 7 OFDM symbols, depending on whether the normal or extended cyclic prefix is employed.

We consider an OFDM system with $K$ users, Let $\Omega{ }_{k}$ denote the index set of subcarriers allocated to user $k$ and $k=1, \ldots, K$. For simplicity, we assume that each subcarrier is occupied by only one user and uniform power allocation across all subcarriers. as [13]:

The channel capacity of a subcarrier in the OFDM multiplex can be expressed

$C_{n}=B_{n} \log _{2}\left(1+\frac{\bar{E}}{T_{g} N_{0} B_{n}} \alpha_{n}^{2}\right)$

where $N_{0}$ is the power spectral density of the additive white Gaussian noise (AWGN) channel (assuming perfect channel estimation), $\alpha_{n}$ is the multipath channel attenuation coefficient of the subcarrier $n$ assumed here as Rayleigh distributed random variable, $T_{s}$ is the symbol time and $\overline{\mathbf{E}}$ is the symbol energy. It has been considered that in an OFDM system the carrier spacing $\left(B_{n}\right)$ is equal to the inverse of the symbol time $\left(T_{s}\right)$.

$C_{n}=B_{n} \log _{2}\left(1+\frac{\bar{E}}{N_{0}} \alpha_{n}^{2}\right)$

$\mathrm{SNR}_{n, k}=\left(\frac{\overline{\mathrm{I}}}{\mathrm{N}_{0}}\right)_{k} \alpha_{n, k}^{2}:$ effective signal-to-noise ratio for the $k$-th user at subcarrier $n$;

$C_{n, k}=B_{n} \log _{2}\left(1+\operatorname{SNR}_{n, k}\right)$

The capacity correction factor $(\mathrm{F})$ is considered here,

$F=\frac{T_{\text {Slot }}-T_{\text {Cpinsot }}}{T_{\text {Slot }}}$ 
$T_{\text {Cpinslot: }}$ The duration of cyclic prefix (CP) in one slot as shown in table 2 .

Table (2) The duration of cyclic prefix for normal and extended cyclic prefix [12].

\begin{tabular}{|c|c|}
\hline CP length & Number of OFDM Symbols/Slot \\
\hline $4.69 \mu \mathrm{s}$ (Normal CP) & 7 OFDM symbols \\
$16.66 \mu \mathrm{s}$ (Extended CP) & 6 OFDM symbols \\
\hline
\end{tabular}

So the capacity of subcarrier is updated to

$C_{n, k}=F B_{n} \log _{2}\left(1+\mathrm{SNR}_{\mathrm{n}, \mathrm{k}}\right)$

The maximum capacity for the $k$-th user is given by:

$R_{k}=\sum_{n \in \Omega_{k}} C_{n, k}$

The resource allocation scheme is design to maximize the system throughput:

$\eta=\sum_{k=1}^{k} R_{k}$

The total channel capacity is not used efficiently at any transmission time if the modulation scheme is fixed. To overcome this drawback, adaptive modulation and coding (AMC) schemes have been introduced [13].

\section{SUBCARRIER ALLOCATION}

The algorithms discussed in this paper are based on the estimation of the channel capacity belonging to the subcarriers. From the previous equations, the channel capacity that a user achieves by the assignment of a certain subcarrier can be determined.

\section{A. Fixed Allocation}

The first allocation algorithm is the fixed allocation algorithm that proposed in [13]. It approximates a uniform capacity distribution, where each user obtains the same number of subcarriers without any consideration to the channel parameters, so the users with the best channel conditions obtain the same number of resources with respect to the users with the worst channel conditions.

Where:

- $S$ : the set of free subcarrier;

- $R_{k}$ : the capacity assigned to the $k$-th user.

1. Initialization:

Set $R_{k}=0, \Omega_{k}=\Phi \quad k=1, \ldots, K$;

2. For $k=1$ to $K$ : 
Assign subcarrier $n$ to user $k$, i.e., add subcarrier $n$ to $\Omega_{k}$. Remove subcarrier $n$ from $S$. Update $R_{k}$ according to (6).

3. Repeat step 2 until $S=\Phi$.

\section{B. Fair Allocation}

To avoid the previous drawback another allocation algorithm is proposed in [13]. The algorithm, assigns the subcarriers to each user depending on the best channel conditions and the channel capacity.

1. Initialization:

a) Set $R_{k}=0, \Omega_{k}=\Phi \quad k=1, \ldots, K$;

For $k=1$ to $K$ :

b) If $5 N R_{n k} \geq S N R_{m k}(m, n \in S)$; assign subcarrier $m$ to user $k$, i.e., add subcarrier $m$ to $\Omega_{k}$. Remove subcarrier $m$ from $S$. Update $R_{k}$ according to (6).

2. Find the user $k$ so that $R_{k} \leq R_{u}$ for each user u; and repeat (1-b) for the corresponding user $k$.

3. Repeat step 2 until $S=\Phi$.

The described algorithm, after an initialization phase, assigns to each user a subcarrier within which the user has the highest SNR. Subsequently all the remaining subcarriers are assigned through an iterative process: the user with the lowest amount of capacity is selected and a subcarrier with the best SNR is allocated to him.

\section{Proposed QoS-Based Allocation Algorithm}

The LTE QoS mechanisms follow a network initiated QoS control based on GBR and non-GBR bearers, which is a class-based packet forwarding treatment for delivering real-time and non-real-time traffic [8].

The previous algorithm may lead to the case where the users occupying air resources do not have a high demand for resources, while other users with urgent traffic demands are not allocated enough resources due to poor channel gains. The proposed algorithm considers the QoS information, e.g., the queuing delay. So we generate a weight value for each user, which is function of the guaranteed QoS parameters as priority, delay and data rate. This is a modification of the existing algorithm in [14].

Then, the system throughput in (7) will be:

I $=\sum_{k=1}^{k} w_{k} R_{k}$

where $W_{k}$ denotes the weight for user $k$ which indicates the QoS information for user $k$. Delay satisfaction indicator $\left(D S_{k}\right)$ indicates the connection delay for user $k$.

$D S_{k}=L_{m}-S_{k}$

where $L_{m}$ is the delay bound for the traffic type, which is the class- $m$ QoS traffic, $S_{k}$ is the waiting time for the data of user $k$, which is the duration between the arriving time and the serving time for the data and neglecting the effect of guard slot.

Let $U_{k, m}$ denote the weight of the data corresponding to subcarrier $n$ of user $k$, is given by: 


$$
U_{k, n}= \begin{cases}{\left[\beta_{m} / D S_{k}\right] \log \left(D_{k, n}+1\right)} & D S_{k}>0 \\ \beta_{m} \log \left(D_{k, n}+1\right) & D S_{k} \leq 0\end{cases}
$$

where $\beta_{m}$ is the class- $m$ QoS coefficient (given in table 1) and $D_{k, n}$ is the amount of data arriving in subcarrier $n$.

So the weight of user $\mathrm{k}$ will be:

$W_{k}=\sum_{n \in \Omega_{k}} U_{k, n}$

The proposed scheduling scheme, assigns a higher weight to the data packets with a less DS, i.e., the data with the least DS should be sent out first.

Letting $R_{k} / W_{k}$ denote the rate-to-weight ratio (RWR), we employ the following proposed subcarrier allocation scheme, where the user with the lowest RWR is allowed to pick subcarriers in each iteration:

1. Initialization:

a) Set $R_{k}=0, \Omega_{k}=\Phi \quad k=1, \ldots, K$;

Sort $W_{k}$ in the descending order,

For $k=1$ to $K$ :

b) If $S N R_{n, k} \geq S N R_{m, k}$ ( $\left.m, n \in S\right)$; assign subcarrier $m$ to user $k$, i.e., add subcarrier $m$ to $\Omega_{k}$. Remove subcarrier $m$ from $S$. Update $R_{k}$ according to (6).

2. Find the minimum $R_{k} / W_{k}(k=1, \ldots, K)$, and repeat (1-b) for the corresponding user $k$.

3. Repeat step 2 until $S=\Phi$.

The proposed algorithm, after an initialization phase, assigns to each user a subcarrier within which the user has the highest SNR. Subsequently all the remaining subcarriers are assigned through an iterative process: the user with the minimum RWR is selected and a subcarrier, which provides the best SNR to that user, is assigned to him.

\section{In the Second Part of this Simulation Scenario:}

LTE system uses resource block (RB) which is the basic unit of exchanging information in both downlink and uplink. The radio resource that is available for a user in the downlink 3GPP LTE system is defined in both frequency and time domains and is called a resource block (RB). In the frequency domain, the RB consists of 12 consecutive subcarriers (180 kHz total bandwidth) and in the time domain it is made up of one time slot of $0.5 \mathrm{~ms}$ duration as shown in fig. (1). We assume that each RB is occupied by only one user.

One resource block contains $N_{S C}^{R E}$ subcarriers; so the channel capacity of a RB in the OFDM multiplex can be expressed as:

$$
C_{m}=N_{S C}^{R E} B_{n} \log _{2}\left(1+\frac{\bar{E}}{T_{z} N_{0} B_{n}} \alpha_{m}^{2}\right)
$$

So eq. (5) is updated to be 
$C_{m, k}=F N_{S C}^{R E} B_{n} \log _{2}\left(1+\mathrm{SNR}_{\mathrm{m}, \mathrm{k}}\right)$

And the same algorithms steps are followed for the RB allocation.

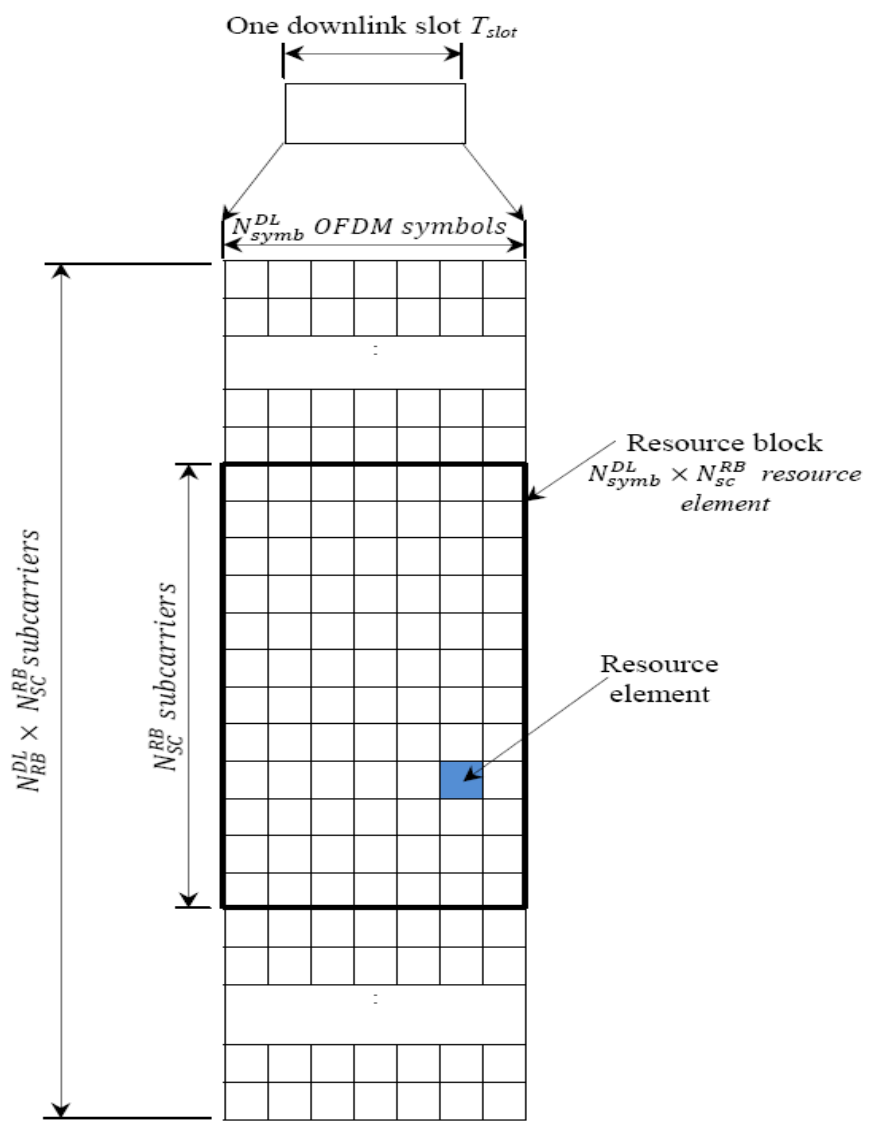

Figure 1. The Downlink time-frequency resource grid [16].

\section{NUMERICAL RESULTS}

In this section, the numerical results is presented to compare the performance of the fixed, fair and proposed scheduling algorithms in LTE downlink direction.

In this simulation, there are 9 users each of them has one type of traffic classes supported by LTE wireless network as in table 1. In our work, we used the normal cyclic prefix.

The values of the system parameters are given in the following table (3):

- For the first part of this paper (subcarrier allocation):

Figure (2) shows the relationship between SNR and the total system capacity. It demonstrates performance of proposed algorithm, compared to fixed and fair allocation schemes. The maximum capacity achieved by the proposed algorithm is much higher than that for fixed scheme and approximately the same as the fair. 
In Fig. (3), the fixed and fair allocation algorithms do not provide any priority for GBR services over Non-GBR. On the other hand, the proposed QoS-based scheduling algorithm provides better capacity for GBR than that for Non-GBR services.

Table (3) The Simulation parameters

\begin{tabular}{|l|l|}
\hline \multicolumn{1}{|c|}{ Parameter } & \multicolumn{1}{|c|}{ Setting } \\
\hline System bandwidth & $10 \mathrm{MHz}$ \\
Number of subcarriers & 1024 \\
Number of occupied subcarriers & 600 \\
Subcarriers per RB $\left(N_{S C}^{F B}\right)$ & 12 \\
RB bandwidth & $180 \mathrm{kHz}$ \\
Number of RBS & 50 \\
Total transmit power & $1 \mathrm{~W}$ \\
Power distribution & Uniform \\
Number of active users & 9 \\
Transmission Time Interval(TTI) & 1 ms \\
Channel type & AWGN channel \\
Channel attenuation coefficient & Rayleigh distributed \\
Modulation/coding rate settings & QPSK : $1 / 2,2 / 3,3 / 4,4 / 5$ \\
& $16 \mathrm{QAM}: 1 / 2,2 / 3,3 / 4,4 / 5$ \\
& 64QAM: $2 / 3,3 / 4,4 / 5$ \\
\hline
\end{tabular}

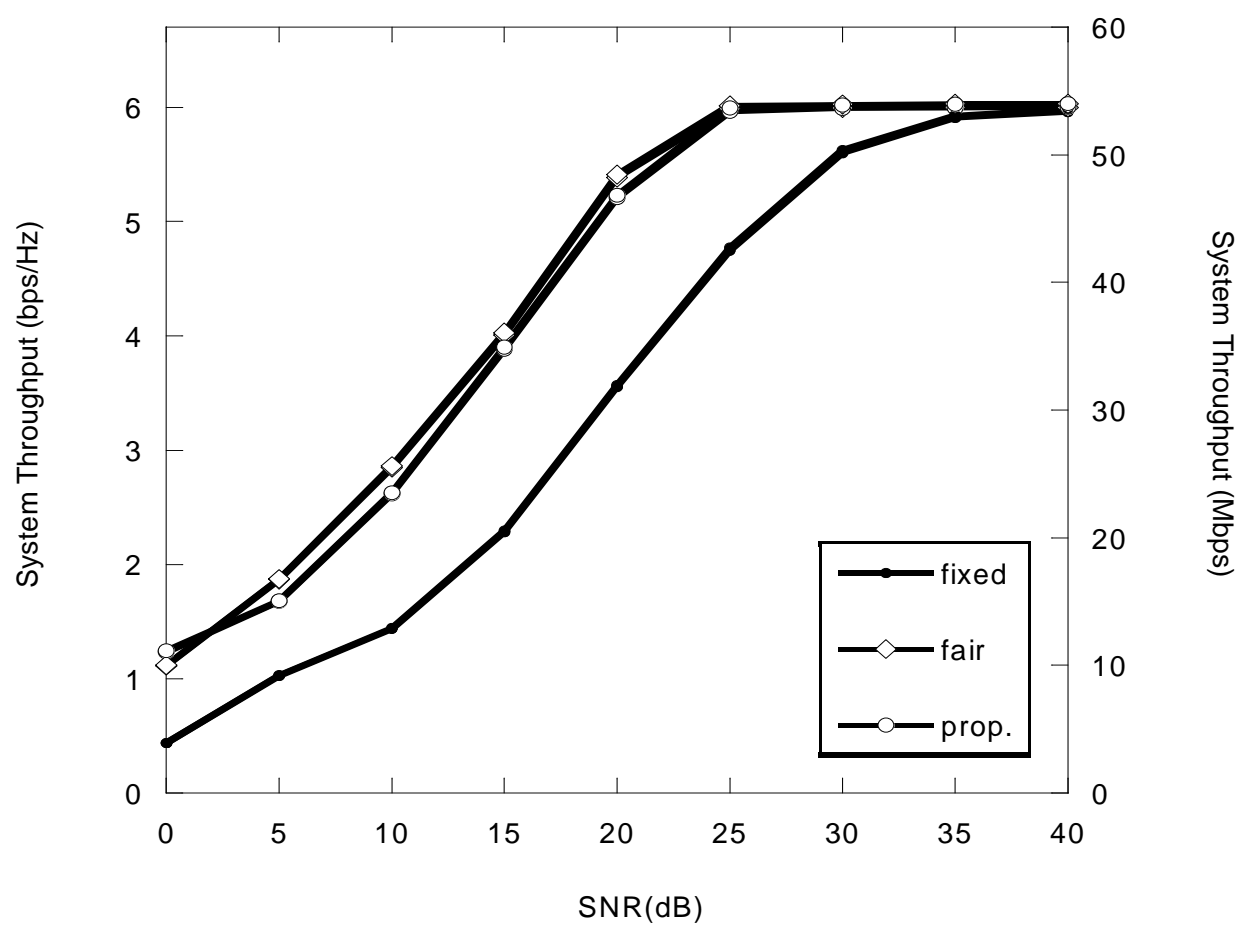


Fig. (2) SNR versus System Throughput in Mbps and bps/Hz for subcarrier allocation.

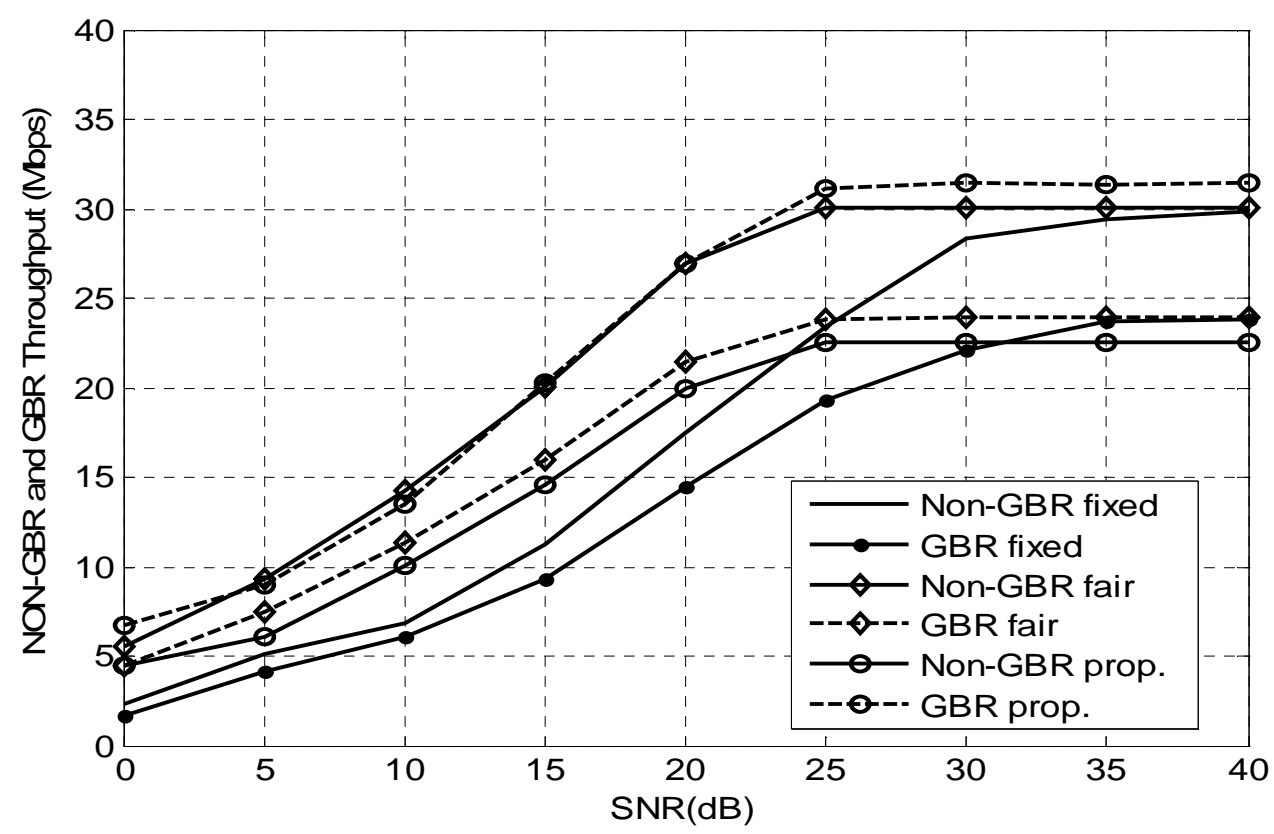

Fig. (3) SNR versus System Throughput in Mbps for Non- GBR and GBR traffic for subcarrier allocation.

- For the second part of this simulation (RB allocation):

Figure (4) demonstrates performance of the proposed QoS-based allocation algorithm, compared to the fixed and fair allocation algorithms in bit per second per hertz and Mbps. The fixed resource allocation scheme achieves low performance while the fair and the proposed scheduling algorithms achieve approximately the same overall capacity for all traffic types. 


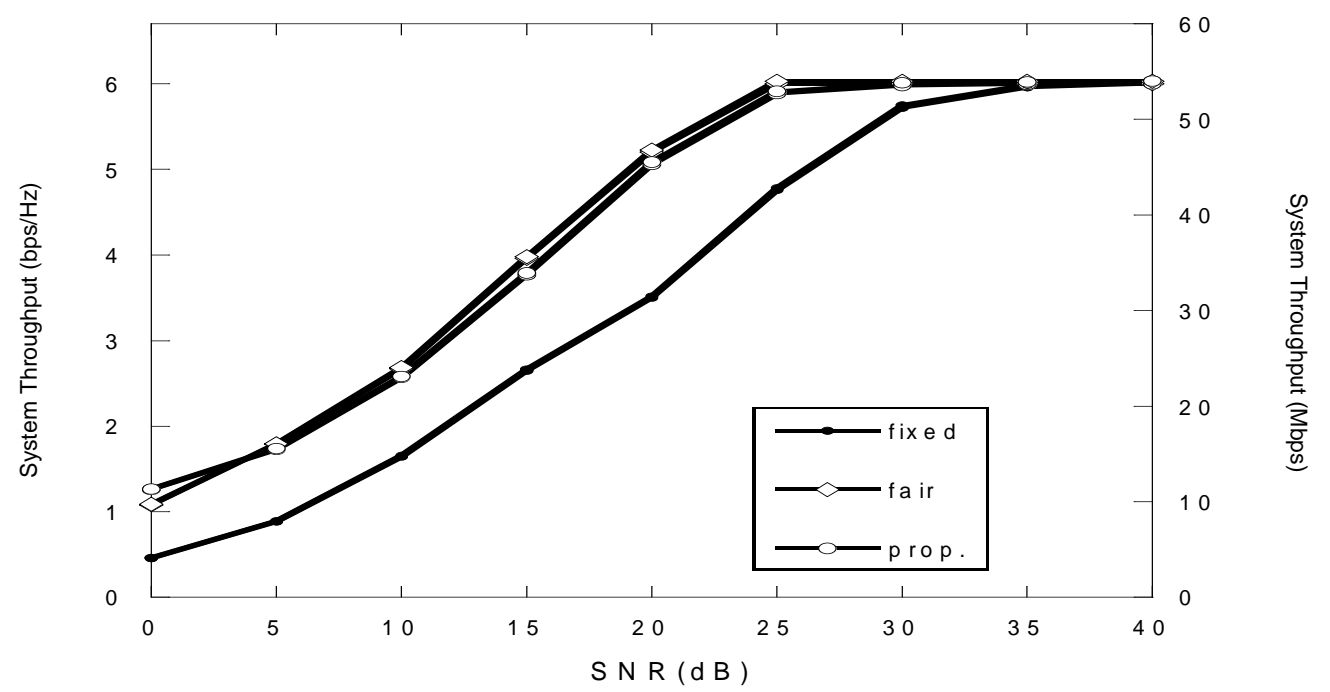

Fig. (4) SNR versus System Throughput in Mbps and bps/Hz for RB allocation.

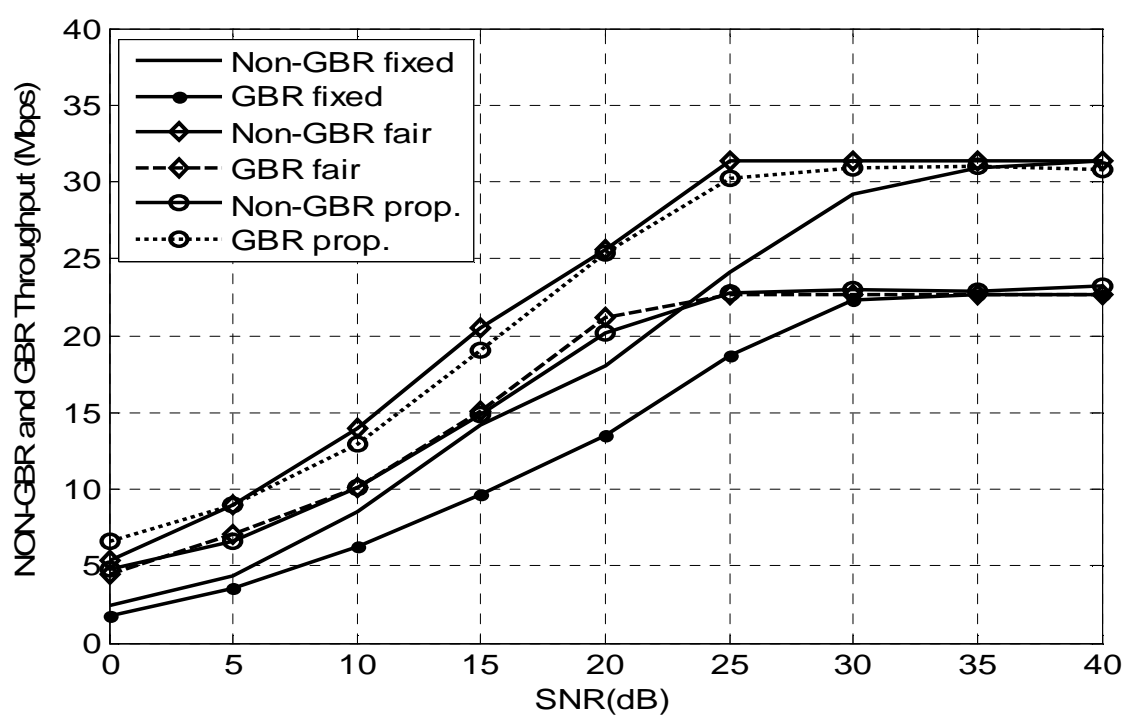

Fig. (5) SNR versus System Throughput in Mbps for Non- GBR and GBR traffic for $\mathrm{RB}$ allocation.

In Fig. (5), the system performance for fixed, fair and the proposed algorithms for the RB allocation. Which when compared to Fig. (3) indicate that, for RB allocation the GBR services can have more capacity than the Non-GBR services in the subcarrier allocation. 


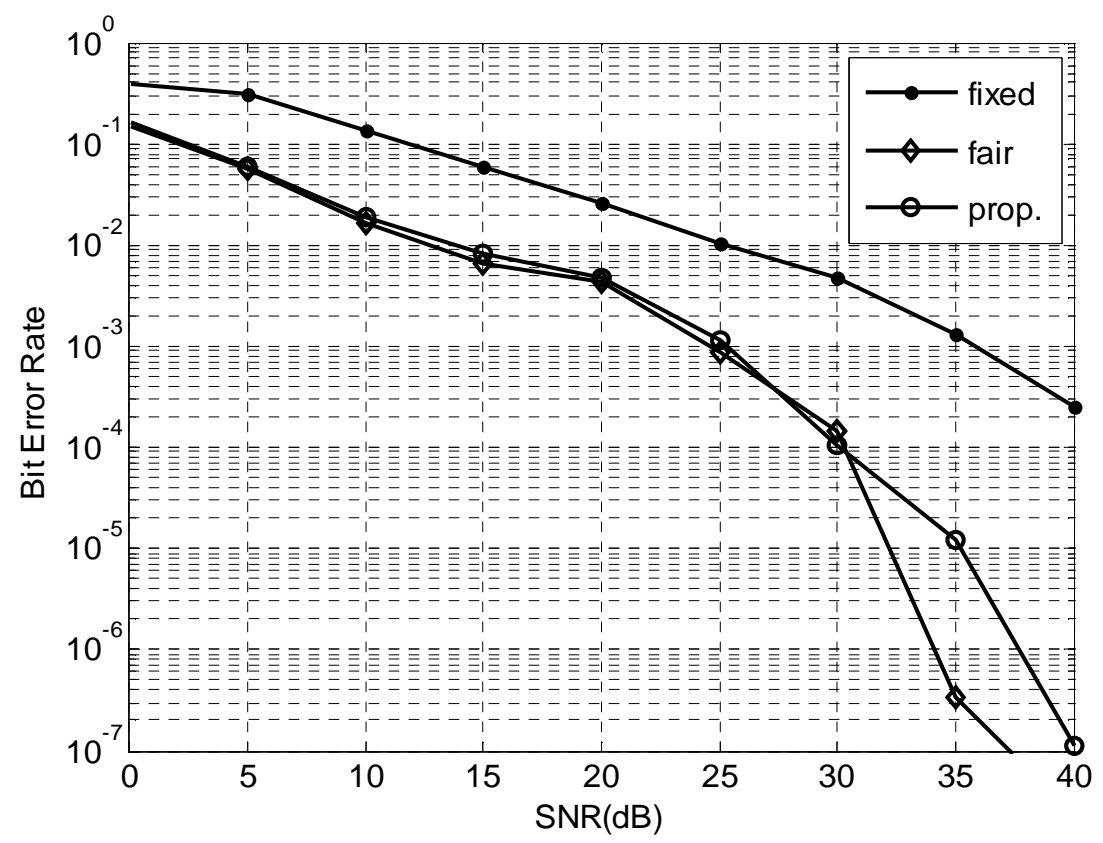

Fig. (6) Bit error rate of the system versus SNR for RB allocation.

Figure 6, points out the better behavior of the proposed algorithm in comparison with the fixed scheduling algorithm in terms of the BER parameter. The figure shows that the proposed algorithm has approximately the same as the fair algorithm.

Through this part of the simulation work, we fix the SNR at $20 \mathrm{~dB}$ and the number of active users varied from 2:74 user divided equally between the GBR and Non-GBR traffic types.

The results in fig. 7 shows that, the total system capacity for the proposed and fair allocation algorithms is much better than that for the fixed allocation algorithm. As we predict; the capacity of the fixed scheme is constant with the increase of user numbers while the capacity of the proposed and the fair schemes are approximately the same and increase with increasing the number of active users.

As indicated from Fig. 8, the fixed and fair scheduling algorithms assign the same amount of capacity for both the GBR and Non-GBR users, so both of them do not provide any priority for GBR services. The proposed algorithm provides high performance for GBR services than Non-GBR services up to 50 users and this is due to the limitation of the resource block number (i.e. $50 \mathrm{RB}$ for $10 \mathrm{MHz}$ bandwidth). 


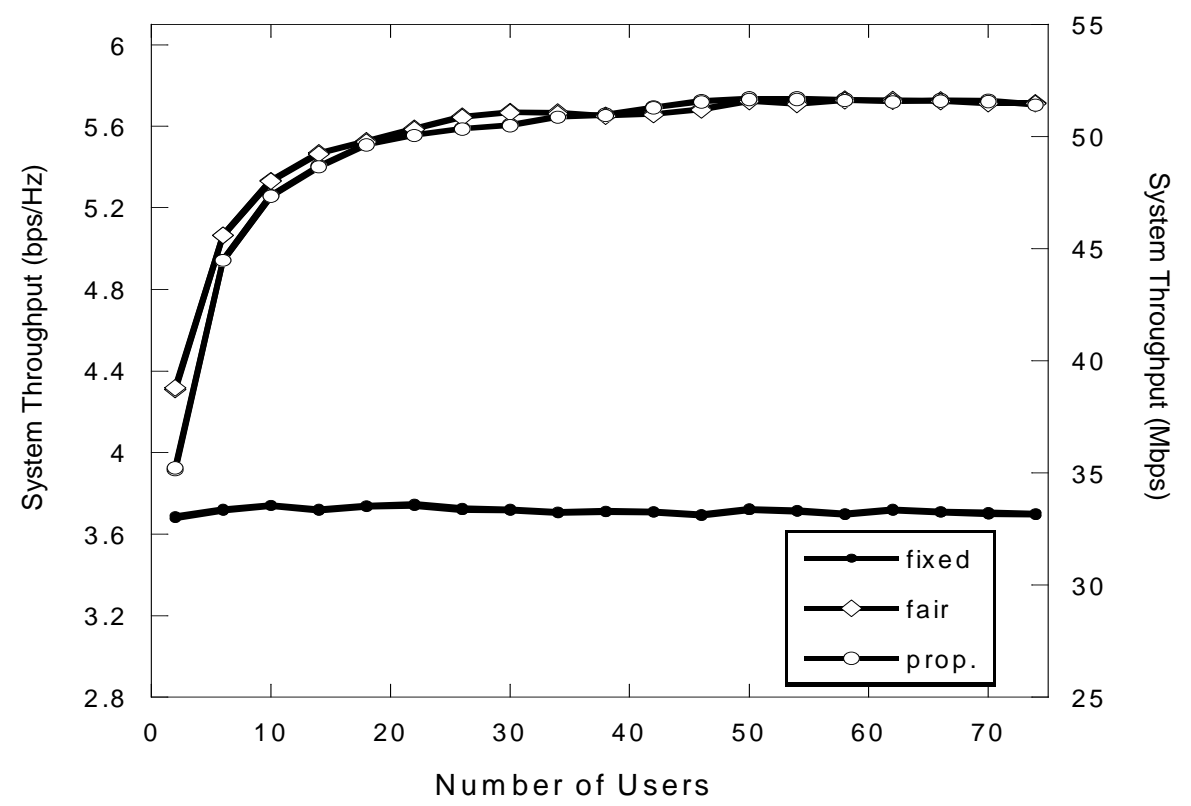

Fig. (7) Throughput in $\mathrm{b} / \mathrm{s} / \mathrm{Hz}$ and Mbps versus Number of users (at SNR=20dB)

\section{CONCLUSION}

In this paper, we have proposed a cross- layer resource allocation scheduling algorithm for downlink LTE network, based on QoS information from the data link layer. And we compare it to the fixed and fair allocation algorithms in two parts. In the first part the allocation algorithms assign individual subcarriers to the users while through the second one, the allocation algorithms assign individual RB to the users. The results show that, the proposed algorithm has approximately the same performance compared to fair allocation algorithm and much better capacity than the fixed schemes. Moreover, it provides a better QoS and performance with guaranteed bit rate services in LTE systems than that of the fixed and fair algorithms.

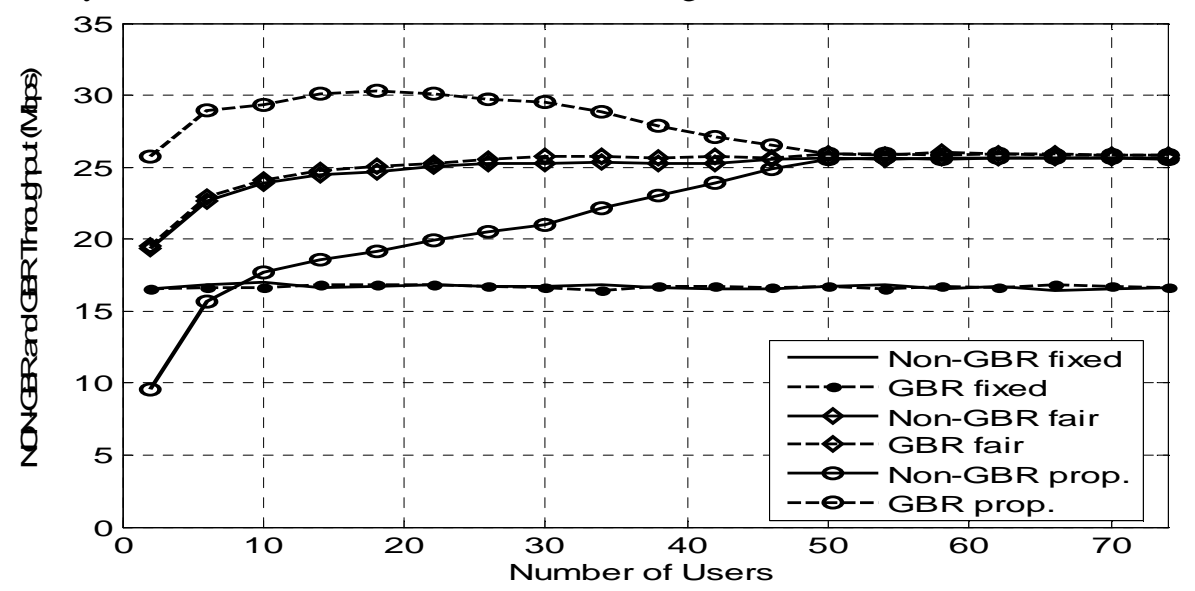


Fig. (8) Non-GBR and GBR System throughput in Mbps versus Number of users $($ at $\mathrm{SNR}=20 \mathrm{~dB})$

\section{REFERENCES}

[1] L. Hanzo, J. Akhtman, L. Wang and M. Jiang., MIMO -OFDM for LTE, WiFi, and WiMAX: coherent versus non-coherent and cooperative turbo-transceivers, John Wiley \& Sons Ltd, Torquay, UK. 2011.

[2] Farooq Khan, LTE for 4G Mobile Broadband Air Interface Technologies and Performance, Cambridge University Press, New York, 2009.

[3] Lingyang Song, Jia Shen, Evolved cellular network planning and optimization for UMTS and LTE, CRC Press Taylor \& Francis Group, LLC, USA, 2011.

[4] Agilent Technologies, "3GPP Long Term Evolution: System Overview, Product Development, and Test Challenges," Application Note, 2008. http://www.lteportal.com/.

[5] Borko Furht and Syed A. Ahson, Long Term Evolution: 3GPP LTE radio and cellular technology, Taylor \& Francis Group, LLC, New York, 2009.

[6] M. Stasiak, M. Głabowski, A. Wisniewski and P. Zwierzykowski, Modeling and dimensioning of mobile networks: from GSM to LTE, John Wiley \& Sons Ltd. 2011.

[7] D. Martín-Sacristán, Jose F. Monserrat, J. Cabrejas-Peňuelas, D. Calabuig, S. Garrigas, and Narcís Cardona, "On the Way towards Fourth-Generation Mobile: 3GPP LTE and LTE-Advanced," EURASIP Journal on Wireless Communications and Networking, Volume 2009, pp. 1 -10, 2009.

[8] M. Alasti, B. Neekzad, Jie Hui and R. Vannithamby, "Quality of Service in WiMAX and LTE Networks," IEEE Communications Magazine, vol. 48, pp. 104 -111, May 2010.

[9] Harri Holma and Antti Toskala, LTE for UMTS: OFDMA and SC-FDMA Based Radio Access, John Wiley \& Sons Ltd. Great Britain, 2009.

[10] Stefania Sesia, Issam Toufik and Matthew Baker, LTE - The UMTS Long Term Evolution: From Theory to Practice, John Wiley \& Sons Ltd. Great Britain, 2009.

[11] F. R. Marques Lima, S. Wanstedt, F. R. Cavalcanti and W. Cruz Freitas Junior, "Scheduling for Improving System Capacity in Multiservice 3GPP LTE," Journal of Electrical and Computer Engineering, Volume 2010, pp. 1 -16, 2010.

[12] QUALCOMM Incorporated, LTE: Overview and Deployment Considerations, 2010, http://www.bandwidthers.com.

[13] Marabissi, D. Tarchi, R. Fantacci, and A. Biagioni, "Adaptive Subcarrier Allocation Algorithms in Wireless OFDMA Systems," IEEE International Conference on Communications - ICC , pp. 3475-3479, 2008.

[14] Nan Zhou, $\mathrm{Xu} \mathrm{Zhu}$ and Yi Huang, "Cross-Layer Optimization with Guaranteed QoS for Wireless Multiuser OFDM Systems," The 18th Annual IEEE International Symposium on Personal, Indoor and Mobile Radio Communications (PIMRC'07), December 2007.

[15] 3GPP TS 23.203 V8.8.0, "Policy and charging control architecture," Dec. 2009. 
[16] 3GPP TS 36.211: "Evolved Universal Terrestrial Radio Access (E-UTRA); Physical Channels and Modulation," V9.1.0, 2010.

[17] Yunzhi Qian, Canjun Ren, Suwen Tang and Ming Chen, "Multi-Service QoS Guaranteed Based Downlink Cross-Layer Resource Block Allocation Algorithm in LTE Systems," International Conference on Wireless Communications \& Signal Processing, WCSP 2009, pp. 1-4, December 2009.

[18] Xiaowei Li, Bingbing Li, Bing Lan, Min Huang and Guanghui Yu, "Adaptive PF Scheduling Algorithm in LTE Cellular System," International Conference on Information and Communication Technology Convergence (ICTC), pp. 501 504, 2010.

[19] Hossam Fattah and Hussein Alnuweiri, "A Cross-Layer Design for Dynamic Resource Block Allocation in 3G Long Term Evolution System," IEEE 6th International Conference on Mobile Adhoc and Sensor Systems, MASS '09. pp. 929 - 934, 2009.

خوارزمية تخصيص الموارد بناءاً علي جودة الخدمة لمنظومة التطوير طويلة المدي (LTE)

تعتبر تقتية شبكات الهاتف المحمول العاملة بنظام ال تي أي تطوراً شاملاً لنظام الجيل الثالث للهاتف المحمول ـ وتقترح هذه الورقة البحثية خوارزم وتخصيص للموارد في شبكات ال تي أي ضامنا لجودة الخدمة وعابرا للطبقات البينية لنموذج الثبكة وخاصة لخدمات المجموعات ـ وقد أوضحت النتائج أن الخوارزم المقترح يمكن أن يؤدي أداء أفضل مـع التطبيقات المتطلبة لوجود جودة الخدمة وذلك عن عدد من الخوارزمات المقترحة سابقاً . 